\title{
A Case of Pott's Puffy Tumor from Odontogenic Source
}

\author{
Tyler Merrill ${ }^{1} \quad$ Kyle Davis $^{1}{ }^{\oplus}$ James Reed Gardner ${ }^{1}$ \\ ${ }^{1}$ Department of Otolaryngology-Head and Neck Surgery, University \\ of Arkansas for Medical Sciences, Little Rock, Arkansas, United States \\ Eur J Gen Dent 2021;10:183-186.
}

\author{
Alissa Kanaan ${ }^{1}(1)$ \\ Address for correspondence Alissa Kanaan, MD, Division of Rhinology \\ and Allergy, Department of Otolaryngology - Head and Neck Surgery, \\ University of Arkansas for Medical Sciences, 4301 W. Markham Street, \\ Slot \#543, Little Rock, AR 72205, United States \\ (e-mail: AKanaan@uams.edu).
}
Abstract
Keywords
- Pott's puffy tumor
- odontogenic
- sinusitis

Pott's puffy tumor (PPT) is a known complication of frontal sinusitis. Odontogenic maxillary sinusitis can seldomly spread to involve the frontal sinus but has not been implicated in the development of PPT. Herein, we describe a case of frontal sinusitis originating from an odontogenic source and culminating in PPT.

\section{Introduction}

First described by Sir Percival Pott in the middle of the 18th century, Pott's puffy tumor (PPT) consists of osteomyelitis of the frontal bone with an associated subperiosteal abscess. ${ }^{1-3}$ Generally, it is seen as a sequela of frontal sinusitis and presents with forehead swelling and pain. Adolescents are at the highest risk for PPT, likely due to increased vascularity of the diploic circulation, ${ }^{1}$ but patients of any age can be affected. This disease entity has become significantly less common since antibiotic therapy became widely available. ${ }^{3}$ Treatment of PPT usually requires a combination of antibiotic therapy and surgery. ${ }^{1,3}$

The recent literature has improved our understanding of odontogenic sinusitis and we now understand that this disease process is much more common than previously thought. ${ }^{4}$ Frontal sinus extension of odontogenic sinusitis occurs relatively frequently, but PPT is remarkably rare.

\section{Case Report}

A 53-year-old male patient presented to the otolaryngology clinic with a 3-month history of yellow nasal discharge, frontal headache, periorbital edema, and progressively worsening midline forehead swelling (-Fig. 1). He was initially evaluated by his primary care provider who obtained computed tomography (CT) imaging and referred the patient to our institution for further evaluation. He was also empirically prescribed an oral antihistamine and nasal steroid spray, which failed to alleviate his frontal headaches. He had no history of recurrent or chronic sinusitis, nasal obstruction, or disruptions in smell or taste, and denied any history of local trauma or prior sinus surgery. His medical history consisted of hypothyroidism, but otherwise, he was healthy without any signs of immunodeficiency. He was prescribed a course of amoxicillin-clavulanate $875-125 \mathrm{mg}$ twice daily, and repeat imaging was obtained prior to surgical intervention.

CT imaging revealed right-sided maxillary, ethmoid, and frontal sinus opacification as well as evidence of PPT (-Figs. 2 and 3). Thickened and sclerosed walls of the bilateral frontal, maxillary, and anterior ethmoid cells were noted, denoting chronic sinus infection. Carious right maxillary molars were also identified with periapical lucency indicating a likely endodontic source of infection (-Fig.4).

He subsequently underwent endoscopic sinus surgery using image-guided navigation. Intraoperatively, copious pus was found in the right maxillary and ethmoid sinuses. When attempting to access the frontal sinus, dense osteitic bone, secondary to chronic inflammation, was encountered. This was subsequently drilled in a DRAF 2a procedure to
DOI https://doi.org/ 10.1055/s-0041-1736376. ISSN 2278-9626.

\footnotetext{
(c) 2021. European Journal of General Dentistry. All rights reserved. This is an open access article published by Thieme under the terms of the Creative Commons Attribution-NonDerivative-NonCommercial-License, permitting copying and reproduction so long as the original work is given appropriate credit. Contents may not be used for commercial purposes, or adapted, remixed, transformed or built upon. (https://creativecommons.org/ licenses/by-nc-nd/4.0/) Thieme Medical and Scientific Publishers Pvt. Ltd., A-12, 2nd Floor, Sector 2, Noida-201301 UP, India
} 


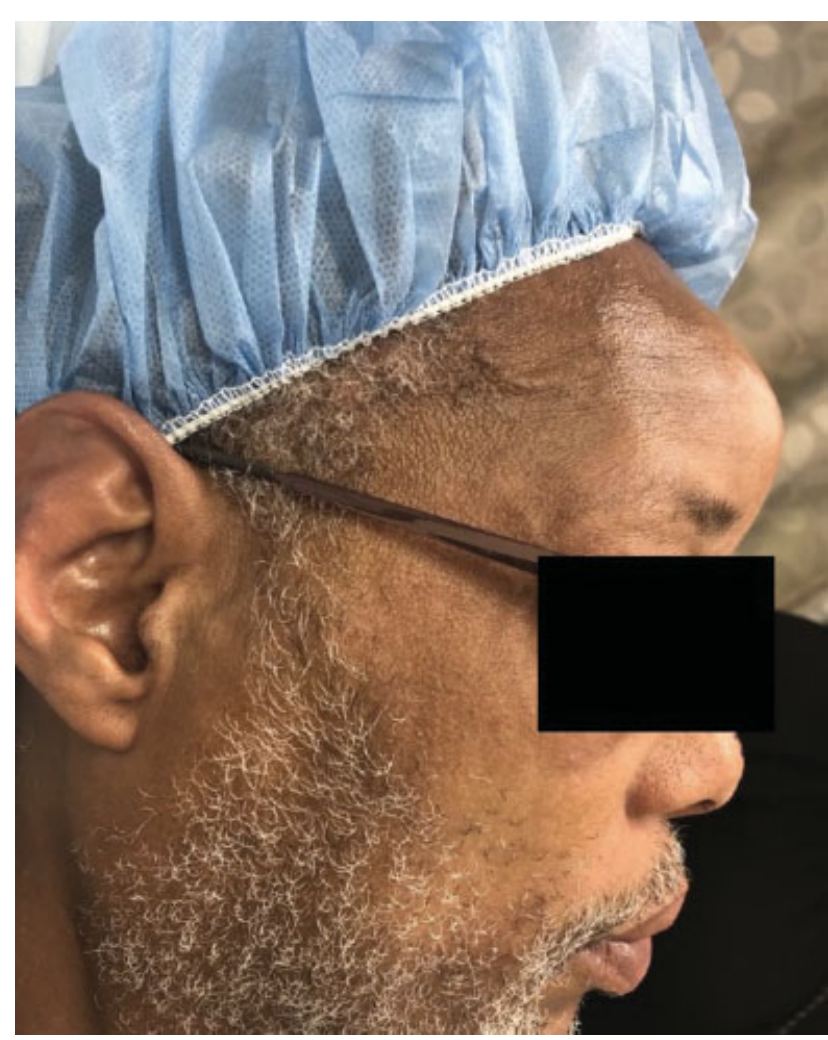

Fig. 1 Preoperative image showing forehead swelling consistent with Pott's puffy tumor.

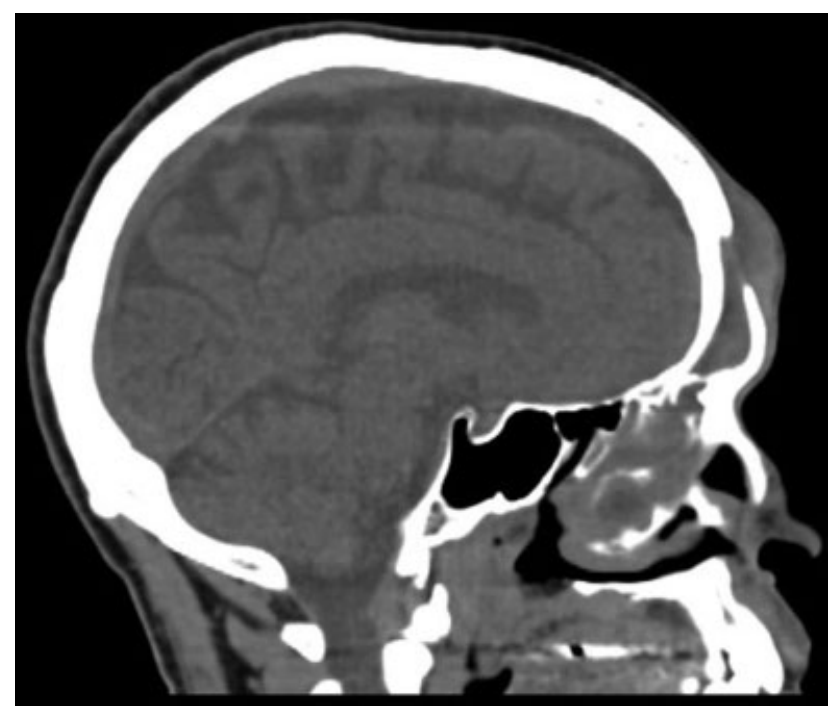

Fig. 2 Sagittal computed tomography (CT) image showing evidence of sinus opacification and Pott's puffy tumor.

access the sinus. Again, a significant volume of purulent debris was encountered and drained endoscopically. All involved sinuses were irrigated, cultures were obtained, and steroid eluting stents were placed. Cultures showed evidence of polymicrobial infection, including Streptococcus salivarius, Prevotella, and Rothia species. After discharge from the hospital, he completed a 6-week course of intravenous vancomycin $15 \mathrm{mg} / \mathrm{kg}$ every 12 hours and piperacillin/tazobactam $3.375 \mathrm{~g}$ every 8 hours.
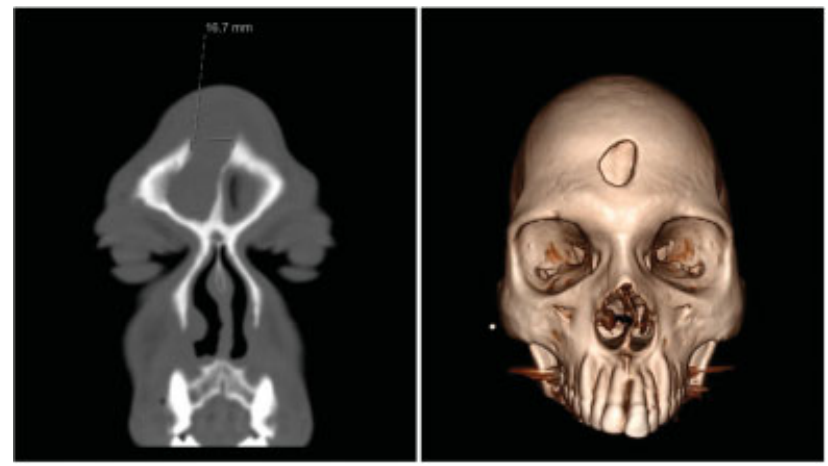

Fig. 3 Coronal computed tomography (CT) image (left) and threedimensional (3D) reconstruction image (right) showing frontal sinus opacification and anterior wall erosion.
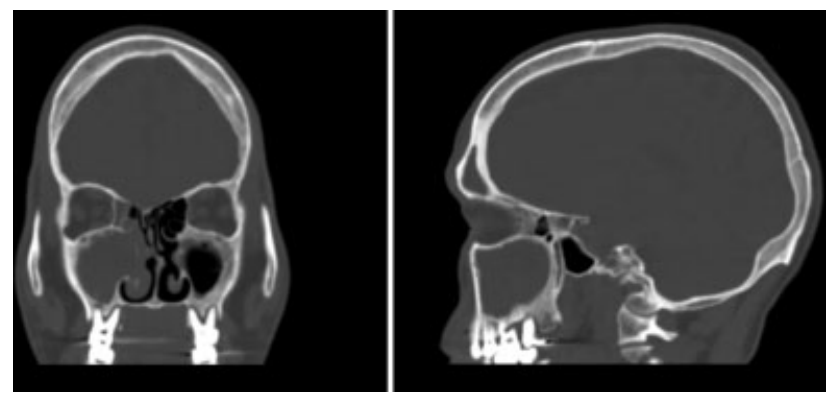

Fig. 4 Coronal computed tomography (CT) image (left) and sagittal $\mathrm{CT}$ image (right) showing complete opacification of the right paranasal sinuses with periapical lucency of the maxillary molars.

He returned for 1-month, 4-month, and 7-month visits postoperatively, with the stents being removed at his 1-month follow-up appointment. His symptoms of headache and drainage had resolved, and the forehead swelling had significantly improved by the first visit. Nasal endoscopy showed a patent frontal sinus with no purulent drainage. Between the 1-month and the 4-month visit, he underwent treatment for dental caries. Imaging at the 7-month appointment showed no evidence of recurrence (-Fig. 5). He was discharged from our clinic at this time having demonstrated lasting resolution of symptoms and no evidence of persistent disease or obstruction on CT or endoscopy.
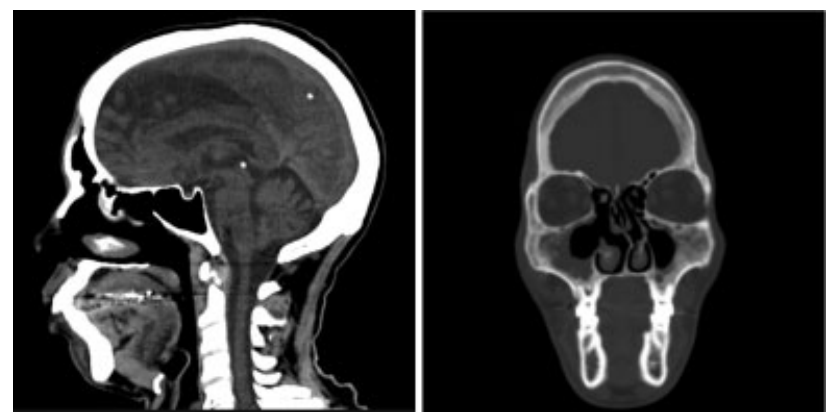

Fig. 5 Seven-month postoperative computed tomography (CT) imaging. Sagittal CT image (left) and coronal CT image (right) showing resolution of PPT and improvement in paranasal sinus disease. 


\section{Discussion}

PPT is a rare disease process in adults. A 2015 review by Tatsumi et al stated that only 54 adult cases had been reported in the Japanese and English literature. ${ }^{1}$ Literature search found odontogenic PPT to be considerably less common with only two cases linking PPT to dental disease. ${ }^{2,3}$

Chandy et al reported a case of PPT that arose following tooth extraction. ${ }^{2}$ In this case, a 21-year-old male patient developed bacteremia, a lung abscess, PPT, and an epidural abscess after undergoing tooth extraction. On presentation, he had no evidence of dental disease, but the sinus disease was attributed to his recent extraction and subsequent bacteremia. Geyton et al described a case in which a retained tooth root served as the likely source of PPT in a 45-year-old male. $^{3}$ The case reported here is singular in terms of presentation of PPT resulting from sinusitis due to routine carious dentition.

Often there are precipitating factors that result in the development of PPT. Adolescents are the most frequently affected population, which is attributed to increased diploic vein flow and maturing of the frontal sinuses. ${ }^{1,2,5}$ The most common cause reported among adults is frontoethmoidal duct stenosis secondary to recurrent sinusitis, trauma, or previous sinus surgery. Chronic rhinosinusitis is a known risk factor for PPT, along with various other underlying diseases and patient factors, including diabetes, chronic renal failure, aplastic anemia, and intranasal cocaine or methamphetamine abuse. ${ }^{1,5}$ In addition, sinus anomalies, such as Kuhn type IV cells, which obstruct the frontal sinus ostium, may increase the risk of developing PPT. ${ }^{5}$

While the patient in our case denied any history of recurrent or chronic sinusitis, we believe that his dental infection likely predisposed him to PPT. CT imaging showed signs of sclerosed and thickened bone, indicating that the patient had chronic inflammation within his paranasal sinuses. Additionally, osteitic bone was encountered during surgery. He likely experienced occult or minimally symptomatic chronic sinusitis, exacerbated by his odontogenic maxillary sinusitis, which spread to the frontal sinuses and progressed to PPT. This patient had no other identifiable risk factors or sinus anomalies that would result in increased susceptibility to PPT.

Odontogenic sinusitis is becoming increasingly recognized as a major source of sinusitis. ${ }^{4}$ Multidisciplinary teams can be involved in the treatment of these patients. Prompt referral of patients with odontogenic sinusitis to dentistry can decrease the morbidity and potential complications from recurrent sinus infections. This is highlighted by the delays in dental care in this case. Classically, unilateral, odontogenic sinusitis can involve any sinus despite only the maxillary sinus being adjacent to the teeth. Frontal sinusitis from an odontogenic origin is a well-known and reported entity. ${ }^{4}$ However, from our literature search, PPT of odontogenic origin is exceptionally rare and therefore noteworthy.

PPT can often be accompanied by intracranial complications. Singh et al found that $85 \%$ of patients with PPT had accompanying intracranial complications. ${ }^{6}$ In these cases, swift diagnosis and treatment are paramount. This case is a reminder that even when patients do not have a history of trauma or chronic sinusitis, but present with symptoms of PPT, prompt imaging and intervention are necessary to mitigate the risk for further morbidity as seen in these previous studies.

\section{Conclusion}

This unusual case of PTT highlights the morbidity that can occur from dental caries and odontogenic sinusitis. Prompt CT imaging in patients reporting symptoms of frontal sinusitis is important to exclude potential extrasinus complications, including PPT. Multidisciplinary care, involving both dentistry and otolaryngology, is vital to provide definitive management in these patients in the form of antibiotic therapy, endoscopic sinus surgery, and dental treatment. Thorough dental examination should be performed in patients presenting with sinusitis and clinicians should encourage patients to undergo immediate dental treatment to prevent recurrences.

Institutional Review Board statement

The Institutional Review Board (IRB) classified this study as exempt.

\section{Authors' Contributions}

Tyler Merrill contributed to data collection and manuscript drafting. Kyle Davis contributed to manuscript drafting. J. Reed Gardner contributed to data collection and manuscript drafting. Alissa Kanaan contributed to data collection and manuscript drafting

\section{Conflict of Interest}

The authors report no personal or financial conflicts of interest and have nothing to declare.

\section{References}

1 Tatsumi S, Ri M, Higashi N, Wakayama N, Matsune S, Tosa M. Pott's puffy tumor in an adult: a case report and review of literature. J Nippon Med Sch 2016;83(05):211-214

2 Chandy B, Todd J, Stucker FJ, Nathan CO. Pott's puffy tumor and epidural abscess arising from dental sepsis: a case report. Laryngoscope 2001;111(10):1732-1734

3 Geyton T, Henderson A, Morris J, McDonald S. A case of Pott's puffy tumour from primary dental infection. BMJ Case Rep 2017; 2017:bcr-2017-222294 doi: 10.1136/bcr-2017-222294

4 Turfe Z, Ahmad A, Peterson EI, Craig JR. Odontogenic sinusitis is a common cause of unilateral sinus disease with maxillary sinus opacification. Int Forum Allergy Rhinol 2019;9(12): $1515-1520$

5 Pendolino AL, Koumpa FS, Zhang H, Leong SC, Andrews PJ. Draf III frontal sinus surgery for the treatment of Pott's puffy tumour in adults: our case series and a review of frontal sinus anatomy risk factors. Eur Arch Otorhinolaryngol 2020;277(08): 2271-2278

6 Singh B, Van Dellen J, Ramjettan S, Maharaj TJ. Sinogenic intracranial complications. J Laryngol Otol 1995;109(10):945-950 